\title{
Accuracy of the Hand-held Wavefront Aberrometer in Measurement of Refractive Error
}

\author{
Jae Yong $\operatorname{Han}^{1}$, Sangchul Yoon ${ }^{1,2}$, Nicolas Scott Brown ${ }^{3}$, Sueng-Han $\operatorname{Han}^{1}$, Jinu $\operatorname{Han}^{1}$ \\ ${ }^{1}$ Institute of Vision Research, Department of Ophthalmology, Yonsei University College of Medicine, Seoul, Korea \\ ${ }^{2}$ Department of Global Health, Graduate School of Public Health, Yonsei University, Seoul, Korea \\ ${ }^{3}$ Ovtiz Corporation, Rochester, NY, USA
}

Purpose: To compare refractive error measured by hand-held wavefront aberrometers with postcycloplegic autorefraction (AR) and cycloplegic refraction (CR).

Methods: The medical records of patients who received refractive measurements using the wavefront aberrometer, postcycloplegic AR, and CR between January 2014 and January 2016 were retrospectively analyzed. The mean differences, 95\% confidence intervals, and limits of agreement (LOA) were calculated for the refractive vector components $\left(M, J_{0}\right.$, and $\left.J_{45}\right)$.

Results: Fifty-one patients $(9.0 \pm 5.5$ years, male $41.2 \%)$ were enrolled in this study, and only the right eye of each was included. Refractive errors ranged from -9.25 to +7.25 diopters (D) for spherical equivalent (median, $0.75 \mathrm{D}$ ). The $M$ component was not significantly different among the three methods $(p=0.080)$. However, the $J_{0}$ vector component was significantly different $(p<0.001)$. After post hoc analysis, the wavefront aberrometer obtained more positive values for $J_{0}$ compared to the other methods. The $J_{45}$ component was not significantly different among the three methods $(p=0.143)$. The mean difference between the wavefront aberrometer and postcycloplegic AR was $-0.115 \mathrm{D}$ (LOA, -1.578 to $1.348 \mathrm{D}$ ) for $M, 0.239 \mathrm{D}$ (LOA, -0.371 to $0.850 \mathrm{D}$ ) for $\mathrm{J}_{0}$, and $-0.015 \mathrm{D}$ (LOA, -0.768 to $0.738 \mathrm{D}$ ) for $J_{45}$. The mean difference between the wavefront aberrometer and $\mathrm{CR}$ was $-0.220 \mathrm{D}$ (LOA, -1.790 to $1.350 \mathrm{D}$ ) for $M, 0.300 \mathrm{D}$ (LOA, -0.526 to $1.127 \mathrm{D})$ for $J_{0}$, and $-0.079 \mathrm{D}(-0.662$ to $0.504 \mathrm{D})$ for $J_{45}$.

Conclusions: The wavefront aberrometer showed good agreement with postcycloplegic AR and CR in spherical equivalents, but tended to produce slightly myopic results. The wavefront aberrometer also overestimated with-the-rule astigmatism. Therefore, we recommend that the device be used for estimations of refractive error, which may be useful for patients who have postural difficulties, live in undeveloped countries, or are bedridden.

Key Words: Corneal wavefront aberration, Mydriatics, Refractive errors, Refractometry

Received: November 12, 2019 Final revision: February 9, 2020

Accepted: March 11, 2020

Corresponding Author: Jinu Han, MD. Institute of Vision Research, Department of Ophthalmology, Yonsei University College of Medicine, 50-1 Yonsei-ro, Seodaemun-gu, Seoul 03722, Korea. Tel: 82-2-2019-3445, Fax: 82-2-3463-1049, E- mail: jinuhan@yuhs.ac
Uncorrected refractive errors are a major cause of amblyopia, which interrupts social functioning and academic performance in children [1]. It is estimated that over one billion people suffer from uncorrected refractive errors worldwide $[2,3]$. The incidence rate of amblyopia caused 

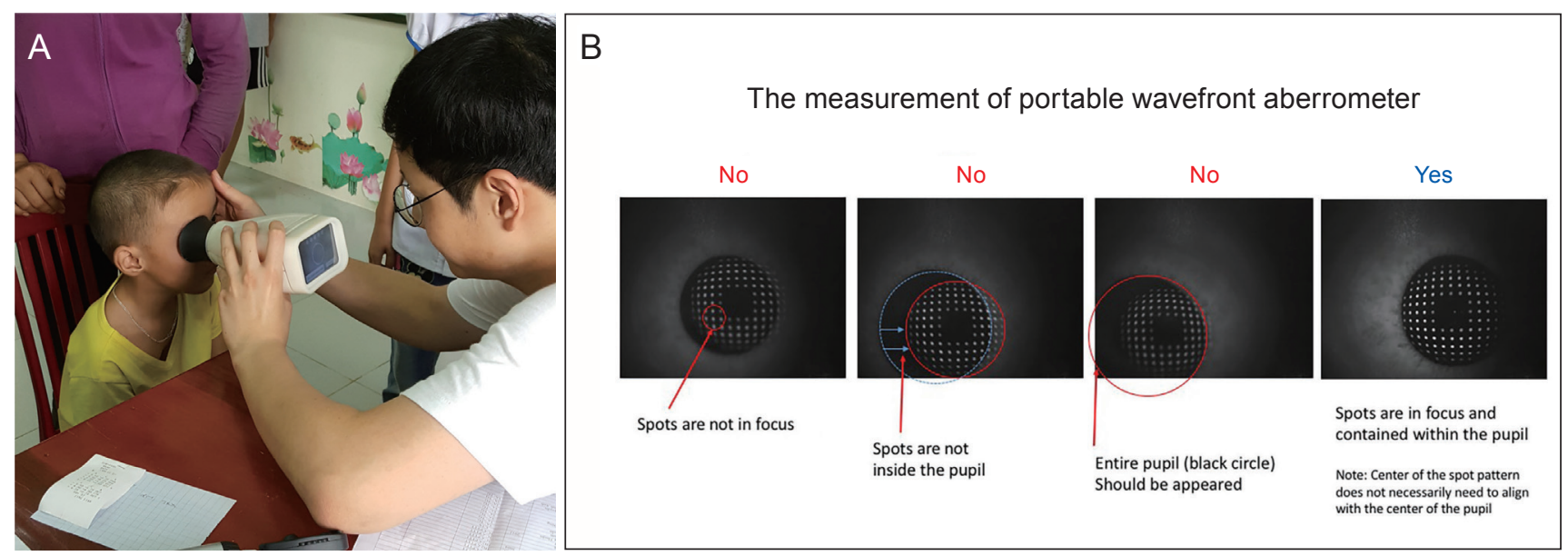

Fig. 1. The measurement of portable wavefront aberrometer. (A) Measurement with the portable OVITZ P10 wavefront aberrometer. (B) Once the device had been positioned properly, the examiner was able to see the array of Hartmann-Shack images on the screen. The array of Hartmann-Shack spots should be clearly focused, and contained within the pupil.

by uncorrected refractive errors is generally higher in low-income or under-developed countries $[4,5]$. With proper diagnosis and early management of refractive errors in children, amblyopia can be treated quickly, which can greatly contribute to improving quality of life. Previous studies have shown that eye screening and correcting refractive errors has the potential to improve social and academic functioning, especially in low-income patients [6,7].

Refractive errors can be measured in several ways, including (1) Objective refraction using an auto kerato-refractometer (autorefraction, AR), (2) cycloplegic refraction (CR) using retinoscopy, or (3) with a wavefront guided aberrometer. $\mathrm{CR}$ is the gold standard for measuring refractive errors, and is still commonly used. However, these procedures have some weaknesses. First, they take a long time and require properly trained optometrists or ophthalmologists. They also show poor test-retest reliability and are easily affected by the technician's ability $[8,9]$. AR is commonly used as a starting point for subjective refraction and is mostly used in settings with high resource availability. In comparison to the other techniques, AR does not require experienced operators, and is faster and easier to perform [10-12]. A wavefront aberrometer is an instrument used to detect the incompleteness of the human optic system at the level below the wavelength of light. Wavefront aberrometer measurements can be categorized according to numerous principles, including Hartmann-Shack, Tscherning, laser ray tracing, and slit sciascopy [13]. Hartmann-Shack sensors are used in ophthalmology to identify visual deficits prior to corneal treatment for complex refractive disorders [8,14]. Unlike objective refraction, subjective manifest refraction attempts to determine refractive errors by trial and error with the patient's cooperation. Through patient interaction, manifest refraction allows clinicians to provide more comfortable eyeglasses for patients. However, it is more time consuming and requires more clinical experience to perform than objective refraction. Additionally, manifest refraction can be inaccurate in patients (especially children) with high accommodative reserve. Therefore, a cycloplegic agent is necessary to relax the accommodative reserve due to the accommodative blur drive which produces myopic over-correction [15-17].

The OVITZ P10 (Ovitz Corporation, Rochester, NY, USA) is a hand-held device used for measuring refractive error using the aberrations of the eye with a Hartmann-Shack wavefront sensor. After acquiring images from wavefront sensing, it uses Zernike decomposition to filter low-order refractive errors from high-frequency aberrations [18]. The device converts Zernike defocus and astigmatism terms into the conventional sphere, cylinder, and axis format. Typically, the device is used for measurement in patients in under-developed countries, or who are bed-ridden or in a wheelchair and may have difficulty with a table-mounted autorefractor (Fig. 1). The aim of this study is to investigate the accuracy of refractive error measurement using a wavefront aberrometer by comparing results to those obtained by postcycloplegic AR and CR. 


\section{Materials and Methods}

The study was conducted at the Severance Hospital, Yonsei University College of Medicine, Seoul, South Korea and was approved by the institutional review board (42019-0743). The study also adhered to the tenets of the Declaration of Helsinki. Written informed consent was waived due to the retrospective nature of the study. The medical records of patients who underwent refractive measurement using a wavefront aberrometer, AR, and CR between January 2014 and January 2016 were retrospectively reviewed. Before starting measurements, patient history, visual acuity, tonometry, slit-lamp examination, fundus examination, and tonometry with a non-contact tonometer were obtained. Patients who had strabismus or refractive errors were included in the study. Patients who had previous corneal or retinal surgery were excluded. Patients who had anterior or posterior segment pathology, including retinopathy or prematurity, were excluded. A total of 51 eyes from 51 patients were analyzed in this study.

Cycloplegia was achieved with three consecutive instillations of $1 \%$ cyclopentolate hydrochloride (OcuCyclo; Samil, Seoul, Korea) and $0.5 \%$ tropicamide with $0.5 \%$ phenylephrine (Mydrin-P; Santen Pharmaceutical, Osaka, Japan), administered 5 minutes apart. The measurement of refractive errors was performed 1 hour after the instillation of the first drop. Adequate cycloplegia was defined as having a subjective amplitude of accommodation less than 2 diopters (D). This was assessed by measuring the pushup amplitude of accommodation with a supplementary $+2.00 \mathrm{D}$ lens added to the distance refractive correction. Subsequently, the refractive error of the subject's eye was assessed in the following order: (1) postcycloplegic AR, (2) CR using retinoscopy, and (3) a hand-held wavefront aberrometer.

Postcycloplegic AR was measured with a table-top auto refractometer (KR-1; Topcon, Tokyo, Japan). First, the patient was seated in front of the on-table auto refractometer, and instructed to place their chin on the chin rest. The physician then ensured that their forehead was touching the forehead rest. After instructing the patient to watch the object on the monitor, the measurement was taken. CR was performed by a single pediatric ophthalmologist for all cases. A trial lens set with retinoscopy was used for CR. The OVITZ P10 wavefront aberrometer was used to obtain five successive readings after cycloplegia, and the values were averaged. When recording refractive error with the OVITZ P10, alignment was achieved by having the subject look into the instrument. The unexamined eye was covered by the patient's hand (Fig. 1A). The patient could see a bright red spot. Once the device had been positioned properly, the examiner was able to see the array of Hartmann-Shack images on the screen (Fig. 1B). When performing measurements, we tried to minimize head or instrument tilting as much as possible. To obtain AR data, the internal software reduced the evaluated pupil size to a diameter of $3 \mathrm{~mm}$.

Refractive error values were obtained from the devices with the conventional notation of sphere (S), minus cylinder form (C), and cylindrical axis $(\alpha)$. All results were converted into power vectors $\left(M, J_{0}\right.$, and $\left.J_{45}\right)$, as described by Liu et al. [19]; spherical equivalent (SE) refraction as $M=$ $\mathrm{S}+\mathrm{C} / 2$, "with-the-rule" and "against-the-rule" astigmatism (Cartesian astigmatism) as $J_{0}=(-\mathrm{C} / 2) \cos (2 \alpha)$, and oblique astigmatism as $J_{45}=(-\mathrm{C} / 2) \sin (2 \alpha)$. In our study, the mean SEs between the right and left eyes were highly correlated (Spearman correlation coefficient $=0.955, p<$ 0.001 ), therefore, only the measurement from the right eye of each patient was analyzed.

Assuming an effect size of 0.25 , type I error of 0.05 , $90 \%$ power, and a non-sphericity correction e 0.8 , the required sample size was 42 subjects using $G$ power 3.1.9.5 (Heinrich-Heine-Universitat, Dusseldorf, Germany) [20]. Statistical analyses were performed using STATA ver. 13.1 (StataCorp., College Station, TX, USA). A $p$-value less than 0.05 was considered statistically significant. Repeated measures analysis of variance (ANOVA) and post hoc analysis using the Tukey test were performed for statistical comparison.

\section{Results}

Among 51 patients, the mean age was $9.0 \pm 5.5$ years, and 21 patients $(41.2 \%)$ were male. The patients had a wide range of refractive errors, ranging from -9.5 to $+7.25 \mathrm{D} \mathrm{SE}$ (median, $0.75 \mathrm{D}$ ), and the mean astigmatism was $-0.69 \mathrm{D}$ (median, -0.5; range, -3 to $0 \mathrm{D}$ ). In addition, 21 patients (31.0\%) had an astigmatism value of at least $-1.00 \mathrm{D}$. The mean values and standard errors of $M, J_{0}$, and $J_{45}$ for the three measurement methods, shown in Table 1, were compared using repeated measures ANOVA. The $M$ value was 
Table 1. Mean values of power vectors measured by three different measurement techniques

\begin{tabular}{llrr}
\hline & $M$ & $J_{0}$ & $J_{45}$ \\
\hline Postcycloplegic autorefraction & $0.16(0.49)$ & $0.37(0.08)$ & $-0.05(0.02)$ \\
Cycloplegic refraction & $0.27(0.47)$ & $0.31(0.56)$ & $0.01(0.02)$ \\
wavefront aberrometer & $0.05(0.53)$ & $0.61(0.08)$ & $-0.07(0.04)$ \\
$p$-value & 0.08 & $<0.001$ & 0.143 \\
\hline
\end{tabular}

Values are presented as vectors (diopters); Values in parentheses indicate 1 standard error of mean.

${ }^{*}$ Calculated from repeated measure analysis of variance.

not significantly different among the three methods ( $p=$ 0.080 , repeated measures ANOVA). The $M$ obtained from the wavefront aberrometer $(0.05 \pm 3.77 \mathrm{D})$ was slightly more myopic than that of $\mathrm{CR}(0.27 \pm 3.34 \mathrm{D}, p=0.064$, post hoc Tukey test). The $J_{0}$ value was significantly different between the three methods $(p<0.001)$. With post hoc analysis, $J_{0}$ between postcycloplegic AR and wavefront aberrometer was significantly significant (mean $J_{0}, 0.37$ vs. $0.61 ; p<0.001$ ), and $J_{0}$ between CR and wavefront aberrometer was also significantly different (mean $J_{0}, 0.31$ vs. $0.61 ; p<0.001)$. There was no significant difference in $J_{0}$ between postcycloplegic AR and CR $(p=0.517)$. The $J_{45}$ component was not significantly different among the three methods ( $p=0.143$ ). With post hoc Tukey analysis, the $J_{45}$ components were not significantly different (postcycloplegic AR vs. CR, $p=0.289$; CR vs. wavefront aberrometer, $p=0.151$; postcycloplegic AR vs. wavefront aberrometer, $p=0.931)$.

In subgroup analysis of astigmatism, vector components in patients who had cylinder values of $0.75 \mathrm{D}$ or larger $(\mathrm{n}=$ 26 ) with $\mathrm{AR}$, the $J_{0}$ value was significantly different between the three methods ( $p=0.001$ ). With post hoc analysis, $J_{0}$ between postcycloplegic AR and wavefront aberrometer was significantly different (mean $J_{0}, 0.70$ vs. 0.95 ; $p=0.047$ ), and $J_{0}$ between CR and wavefront aberrometer was also significantly different (mean $J_{0}, 0.54$ vs. $0.95 ; p<$ 0.001). There was no significant difference in $J_{0}$ between postcycloplegic AR and CR $(p=0.229)$. The $J_{45}$ component was not significantly different among the three methods ( $p$ $=0.225$ ). The mean difference of $M$ between the wavefront aberrometer and postcycloplegic AR was $-0.115 \mathrm{D}$.

To compare the differences between $\mathrm{AR}, \mathrm{CR}$, and the wavefront aberrometer, the $95 \%$ limits of agreement (LOA) were quantified using the technique described by Bland and Altman [21]. The difference between each vector mea- surement with respect to the wavefront aberrometer was determined, and the LOA was calculated as 1.96 multiplied by the standard deviation of the differences [21]. Between the wavefront aberrometer and postcycloplegic AR, the mean $M$ difference was $-0.115 \mathrm{D}$, and the LOA for $M$ was -1.578 to 1.348 . For $J_{0}$, the mean difference was $0.239 \mathrm{D}$, and the LOA was -0.371 to 0.850 . For $J_{45}$, the mean difference was $-0.015 \mathrm{D}$, and the LOA was -0.768 to 0.738 . Between the wavefront and $\mathrm{CR}$, the mean $M$ difference was $-0.220 \mathrm{D}$, and the LOA was -1.790 to 1.350 . The mean difference was $0.300 \mathrm{D}$ and the LOA was -0.526 to 1.127 for $J_{0}$. The mean difference for $J_{45}$ was $-0.079 \mathrm{D}$ and the LOA was -0.662 to 0.504 (Fig. 2A-2F).

The axes of the cylindrical components measured using the wavefront aberrometer were compared to AR and CR in patients with cylinder $0.75 \mathrm{D}$ or more $(\mathrm{n}=26)$. Compared to AR, only $46.2 \%$ of the cylinders were within $5.0^{\circ}$; $61.5 \%$ were within $10^{\circ}$, and $76.9 \%$ were within $20^{\circ}$. Compared to $\mathrm{CR}, 61.5 \%$ were within $5.0^{\circ}, 76.9 \%$ were within $10^{\circ}$, and $96.2 \%$ were within $20^{\circ}$ (Table 2 ).

\section{Discussion}

In this study, we investigated the accuracy of a handheld wavefront aberrometer for measuring refractive errors compared to conventional methods including AR and CR. Our results indicate that the $M$ values were not significantly different among the three methods. The wavefront aberrometer showed a slight myopic shift compared to the other methods, but the difference was not statistically significant. $J_{0}$ refers to cylinder power set at orthogonally $90^{\circ}$ and $180^{\circ}$ meridians, representing Cartesian astigmatism [19]. The vector values in $J_{0}$ tended to be more positive in measurements made with the wavefront aberrometer $(p<$ 
A

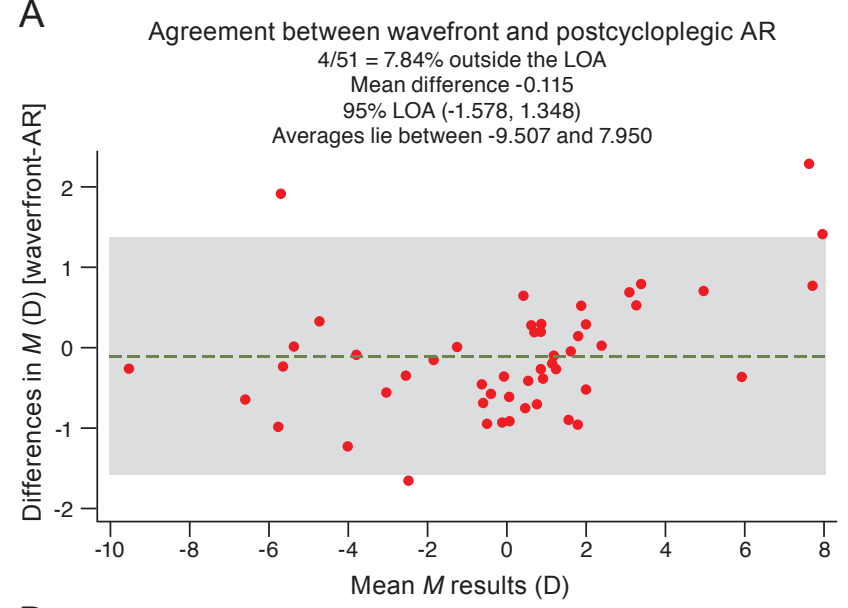

B

B Agreement between wavefront and postcycloplegic AR

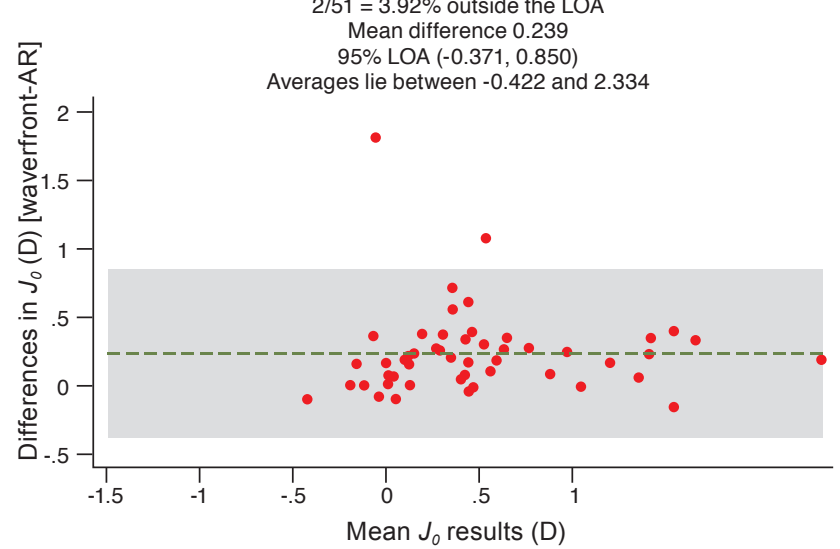

C

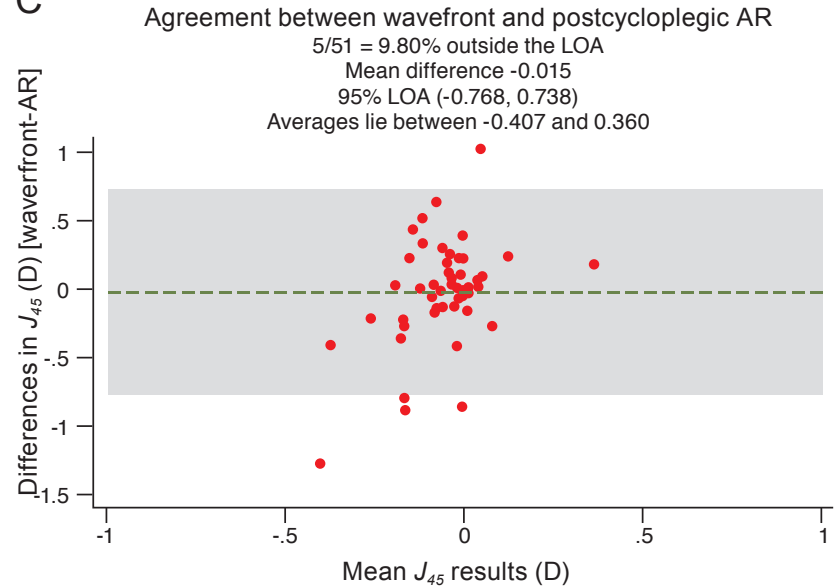

D

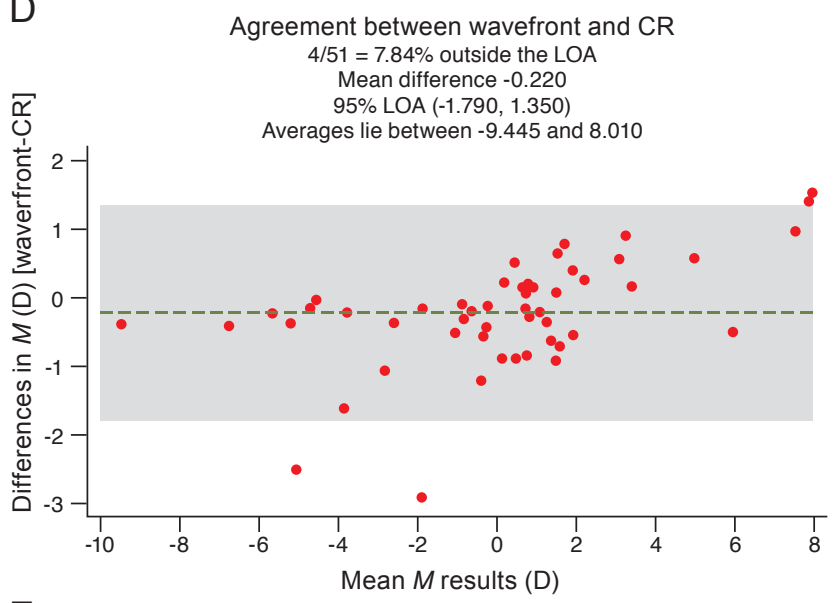

E
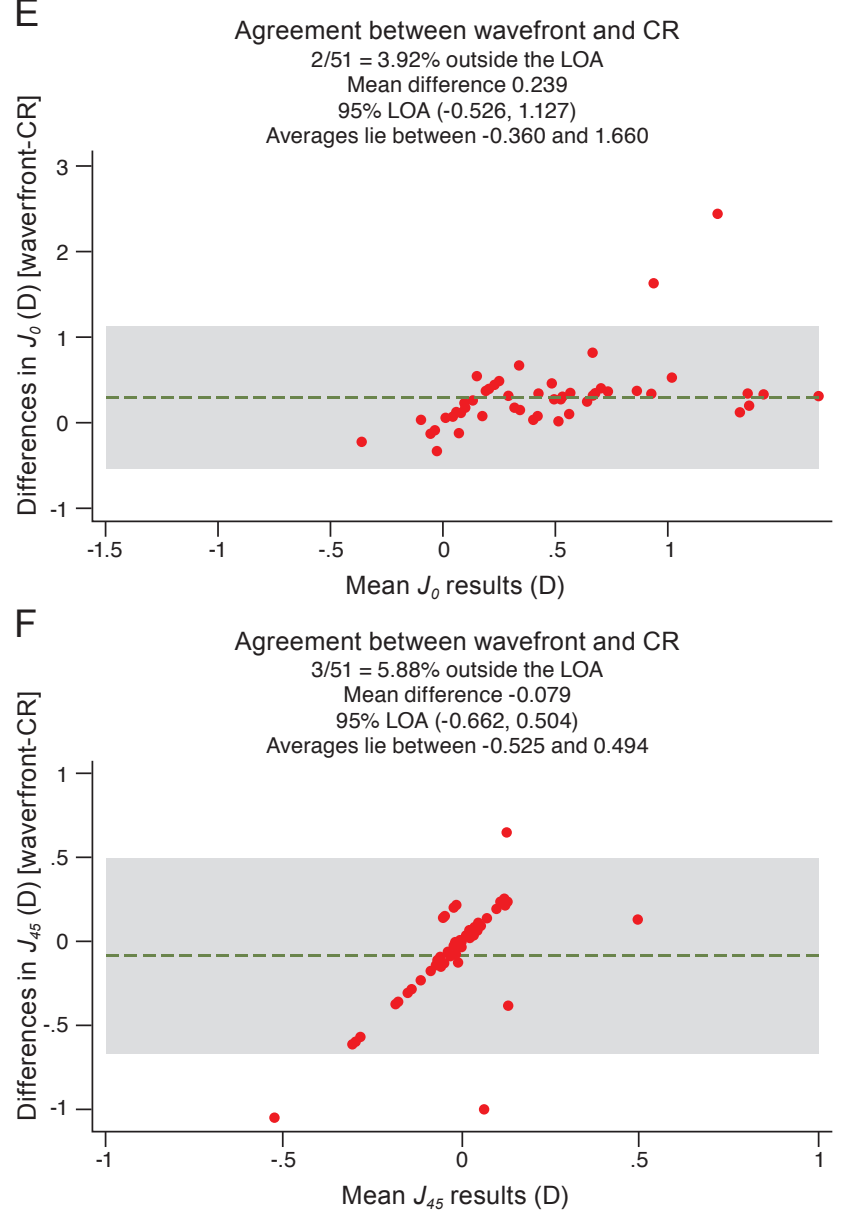

Fig. 2. Bland-Altman plot showing the differences between wavefront aberrometer and postcycloplegic autorefraction (AR) (left), wavefront aberrometer and cycloplegic refraction (CR) (right) in 51 patients at each power vectors $\left(M, J_{0}, J_{45}\right)$. Agreement between the wavefront aberrometer and postcycloplegic AR, (A) the mean $M$ difference was -0.115 diopters (D), and the limits of agreement (LOA) was -1.578 to 1.348 . (B) For $J_{0}$, the mean difference was $0.239 \mathrm{D}$, and the LOA was -0.371 to 0.850 . (C) For $J_{45}$, the mean difference was $-0.015 \mathrm{D}$, and the LOA was -0.768 to 0.738 . Between the wavefront and CR, (D) the mean $M$ difference was $-0.220 \mathrm{D}$, and the LOA was -1.790 to 1.350 . (E) The mean difference was $0.300 \mathrm{D}$ and the LOA was -0.526 to 1.127 for $J_{0}$. (F) The mean difference for $J_{45}$ was $-0.079 \mathrm{D}$ and the LOA was -0.662 to 0.504 . 
Table 2. Comparison of axes of the cylindrical components between wavefront aberrometer, postcycloplegic AR, and CR in subjects with cylinder 0.75 diopters or more with AR $(\mathrm{n}=$ 26)

\begin{tabular}{lcc}
\hline Difference in axis & $\begin{array}{c}\text { Wavefront } \\
\text { aberrometer and } \\
\text { postcycloplegic AR }\end{array}$ & $\begin{array}{c}\text { Wavefront } \\
\text { aberrometer and } \\
\text { CR }\end{array}$ \\
\hline Axis $\leq \pm 5^{\circ}$ & $12(46.2)$ & $16(61.5)$ \\
$\pm 5^{\circ}<$ axis $\leq \pm 10^{\circ}$ & $4(15.4)$ & $4(15.4)$ \\
$\pm 10^{\circ}<$ axis $\leq \pm 20^{\circ}$ & $4(15.4)$ & $5(19.2)$ \\
Axis $> \pm 20^{\circ}$ & $6(23.0)$ & $1(3.8)$ \\
\hline
\end{tabular}

Values are presented as number (\%).

$\mathrm{AR}=$ autorefraction; $\mathrm{CR}=$ cycloplegic refraction .

0.001 ), indicating the trend toward with-the-rule astigmatism. This trend was also observed in patients who had cylinder $0.75 \mathrm{D}$ or more. $J_{45}$ refers to a cross-cylinder set at $45^{\circ}$ and $135^{\circ}$, representing oblique astigmatism. The oblique astigmatism component was not significantly different among the three methods. Because our subjects were children, they usually had with-the-rule astigmatism. As the wavefront aberrometer showed slight myopic shift in SE, the astigmatism component presented by minus cylinder is also expected to be overestimated.

Previous studies compared refractive error measurements between hand-held or table mounted AR, wavefront aberrometers, and CR. Liang et al. [22] compared handheld and table mounted AR in both cycloplegic and non-cycloplegic conditions. With hand-held AR, the mean myopic bias was $0.59 \mathrm{D}$ under non-cycloplegic conditions. However, there were no significant differences between hand-held and table-mounted AR under cycloplegic conditions. Iuorno at el. [23] compared hand-held AR under non-cycloplegic conditions to $\mathrm{CR}$ with retinoscopy in 91 children. They reported that hand-held AR results were more myopic than CR. Although all refractive measurements were taken after cycloplegia, some residual accommodation might have remained. The other eye was occluded during wavefront aberrometer measurement, and the subject was required to look into the instrument. Therefore, this might explain the slight myopic shift in SE with the wavefront aberrometer.

Previous studies also compared wavefront aberrometers with AR and subjective refraction. Early wavefront aberrometers, such as the complete ophthalmic analysis system, were reported to have similar accuracy to AR [24-26].
Pesudovs et al. [27] compared the complete ophthalmic analysis system aberrometer with AR and subjective refraction, and found significant levels of similarity between the three devices. Bennett et al. [28] compared the wavefront aberrometer based on dynamic sciascopy to AR and subjective refraction. AR and the wavefront aberrometer showed agreement with subjective refraction. Although AR showed slightly higher levels of agreement than the wavefront aberrometer in their study, the difference was not significant. The table-mounted wavefront aberrometer has also been compared with CR. Fernandez de Castro et al. [29] compared CR with three different wavefront aberrometers based on the Hartmann-Shack principle in 55 subjects. Although there were small differences between the three devices, all of the devices correlated well with CR in this study.

In our study, the hand-held wavefront aberrometer showed good agreement with low bias in $M(-0.115 \mathrm{D}$ with AR, $-0.220 \mathrm{D}$ with CR). Unlike $M$, the cylinder measurements were significantly different. The bias was $-0.239 \mathrm{D}$ with $\mathrm{AR}$ and $0.300 \mathrm{D}$ with $\mathrm{CR}$ in the $J_{0}$ component, showing significant differences in Cartesian astigmatism. In cases of oblique astigmatism, the bias was nearly absent for both AR (-0.015 D) and CR (-0.079 D). Because the number of cases with oblique astigmatism component was small in our cohort, analysis of $J_{45}$ is clinically meaningless (Fig. 2). In patients who had cylinder $0.75 \mathrm{D}$ or more, the axis differences were relatively small. Only $23.0 \%$ and $3.8 \%$ of patients were outside $20^{\circ}$ compared to AR and $\mathrm{CR}$, respectively (Table 2). The relative difference of the astigmatism axis may be attributed to the following reasons: first, the wavefront aberrometer used in this study is a hand-held device, which makes it more vulnerable to misalignment during measurement. Similarly, shaking of the operator's hand during measurement may also contribute to inaccuracy. Finally, most of our subjects were children, so head tilting during measurement could explain poor agreement of astigmatism component. Third, ophthalmologists tend to do measurements at $180^{\circ}$ or $170^{\circ}$ rather than $175^{\circ}$ or $173^{\circ}$.

Our study has some limitations. First, the sample size was relatively small, and most of the participants were young children. Older children or adults may have higher levels of agreement with CR. Second, none of the participants had other ophthalmic diseases. Thus, we could not determine the accuracy of the hand-held wavefront aber- 
rometer in various clinical situations, such as cataract, corneal, and retinal disorders. Moreover, we measured the refractive errors once using the wavefront aberrometer, thus, it was not possible to test repeatability. Further prospective studies with larger sample sizes are needed to confirm wavefront aberrometer repeatability.

In conclusion, the hand-held wavefront aberrometer offers reliable information regarding refractive errors. Measurements made with the device did not differ significantly from those obtained by postcycloplegic AR and CR, especially in SE. However, the wavefront aberrometer showed significant differences in $J_{0}$ vector component. Therefore, possible errors in cylinder measurement should be considered when measuring refractive errors using the hand-held wavefront aberrometer. This device uses wave-front technology when measuring the refractive error, and the OVITZ P10 $(0.7 \mathrm{~kg})$ is smaller and lighter than a spot vision screener $(3.232 \mathrm{~kg})$ and plusoptiX S12 $(1.1 \mathrm{~kg})$. It is not restricted by space, and is less affected by the patient's position. This is especially helpful for patients who have ambulation or postural disabilities that make traditional measurements difficult to obtain. Moreover, this device may be a good tool for screening large numbers of children at their schools with low cost and minimal training. Such examinations could trigger referral to ophthalmologists for actual eyeglass prescriptions, and thereby overall improve early detection of refractive errors in young children. Further, the device may be useful for physicians in low-income countries where medical space is limited.

\section{Conflict of Interest}

No potential conflict of interest relevant to this article was reported.

\section{Acknowledgements}

The authors would like to extend our thanks to Felix Kim for providing the OVITZ P10 device. We also thank Geunyoung Yoon at University of Rochester for scientific advice.

\section{References}

1. Griffith JF, Wilson R, Cimino HC, et al. The use of a mobile van for school vision screening: results of 63 841 evaluations. Am J Ophthalmol 2016;163:108-14.

2. Durr NJ, Dave SR, Lage E, et al. From unseen to seen: tackling the global burden of uncorrected refractive errors. Annu Rev Biomed Eng 2014;16:131-53.

3. Resnikoff S, Pascolini D, Mariotti SP, Pokharel GP. Global magnitude of visual impairment caused by uncorrected refractive errors in 2004. Bull World Health Organ 2008;86:63-70.

4. Ruderman M. Children's vision and eye health: a snapshot of current national issues. 1st ed. Chicago: National Center for Children's Vision and Eye Health at Prevent Blindness; 2016. p. 7-12.

5. Multi-Ethnic Pediatric Eye Disease Study (MEPEDS) Group. Prevalence and causes of visual impairment in African-American and Hispanic preschool children: the multi-ethnic pediatric eye disease study. Ophthalmology 2009;116:1990-2000.

6. Mayro EL, Hark LA, Shiuey E, et al. Prevalence of uncorrected refractive errors among school-age children in the School District of Philadelphia. $J$ AAPOS 2018;22:214-7.

7. Dirani M, Zhang X, Goh LK, et al. The role of vision in academic school performance. Ophthalmic Epidemiol 2010;17:18-24.

8. Hervella L, Villegas EA, Prieto PM, Artal P. Assessment of subjective refraction with a clinical adaptive optics visual simulator. J Cataract Refract Surg 2019;45:87-93.

9. Zadnik K, Mutti DO, Adams AJ. The repeatability of measurement of the ocular components. Invest Ophthalmol Vis Sci 1992;33:2325-33.

10. Sun JK, Qin H, Aiello LP, et al. Evaluation of visual acuity measurements after autorefraction vs manual refraction in eyes with and without diabetic macular edema. Arch Ophthalmol 2012;130:470-9.

11. Bullimore MA, Adams CW, Fusaro RE, et al. Patient acceptance of auto-refractor and clinician prescriptions: a randomized clinical trial. Vis Sci Appl 1996;1:194-7.

12. Durr NJ, Dave SR, Lim D, et al. Quality of eyeglass prescriptions from a low-cost wavefront autorefractor evaluated in rural India: results of a 708-participant field study. BMJ Open Ophthalmol 2019;4:e000225.

13. Visser N, Berendschot TT, Verbakel F, et al. Evaluation of 
the comparability and repeatability of four wavefront aberrometers. Invest Ophthalmol Vis Sci 2011;52:1302-11.

14. Maeda N, Fujikado T, Kuroda T, et al. Wavefront aberrations measured with Hartmann-Shack sensor in patients with keratoconus. Ophthalmology 2002;109:1996-2003.

15. Vasudevan B, Ciuffreda KJ, Meehan K, et al. Comparison of objective refraction in darkness to cycloplegic refraction: a pilot study. Clin Exp Optom 2016;99:168-72.

16. Cordonnier M, Kallay O. Non-cycloplegic screening for refractive errors in children with the hand-held autorefractor Retinomax: final results and comparison with non-cycloplegic photoscreening. Strabismus 2001;9:59-70.

17. Rosenfield M, Chiu NN. Repeatability of subjective and objective refraction. Optom Vis Sci 1995;72:577-9.

18. Guirao A, Williams DR. A method to predict refractive errors from wave aberration data. Optom Vis Sci 2003;80:3642.

19. Liu YC, Chou P, Wojciechowski R, et al. Power vector analysis of refractive, corneal, and internal astigmatism in an elderly Chinese population: the Shihpai Eye Study. Invest Ophthalmol Vis Sci 2011;52:9651-7.

20. Cohen J. A power primer. Psychol Bull 1992;112:155-9.

21. Bland JM, Altman DG. Statistical methods for assessing agreement between two methods of clinical measurement. Lancet 1986;1:307-10.

22. Liang CL, Hung KS, Park N, et al. Comparison of measurements of refractive errors between the hand-held Retinomax and on-table autorefractors in cyclopleged and noncyclopleged children. Am J Ophthalmol 2003;136:1120-8.

23. Iuorno JD, Grant WD, Noel LP. Clinical comparison of the Welch Allyn SureSight handheld autorefractor versus cycloplegic autorefraction and retinoscopic refraction. $J A A$ POS 2004;8:123-7.

24. Martinez AA, Pandian A, Sankaridurg P, et al. Comparison of aberrometer and autorefractor measures of refractive error in children. Optom Vis Sci 2006;83:811-7.

25. Salmon TO, van de Pol C. Evaluation of a clinical aberrometer for lower-order accuracy and repeatability, higher-order repeatability, and instrument myopia. Optometry 2005;76:461-72.

26. Cheng X, Himebaugh NL, Kollbaum PS, et al. Validation of a clinical Shack-Hartmann aberrometer. Optom Vis Sci 2003;80:587-95.

27. Pesudovs K, Parker KE, Cheng H, Applegate RA. The precision of wavefront refraction compared to subjective refraction and autorefraction. Optom Vis Sci 2007;84:387-92.

28. Bennett JR, Stalboerger GM, Hodge DO, Schornack MM. Comparison of refractive assessment by wavefront aberrometry, autorefraction, and subjective refraction. J Optom 2015;8:109-15.

29. Fernandez de Castro LE, Sandoval HP, Al Sarraf O, et al. Relationship between cycloplegic and wavefront-derived refraction. J Refract Surg 2003;19:S677-81. 